\title{
Disinvestment from low value clinical interventions: NICEly done?
}

In this Analysis article by Sarah Garner and Peter Littlejohns (BMJ 2011;343:d4519, doi:10.1136/bmj.d4519), Garner would like to add that she is a 2010-11 Commonwealth Fund Harkness fellow in healthcare policy and practice. 